\title{
Infrared Spectrometer, Technology and Applications
}

\author{
Arno Simon, Stephan Lüttjohann \\ Bruker Optics GmbH \\ Rudolf-Plank-Str. 27, 76275 Ettlingen, Germany
}

Infrared Spectroscopy started in the beginning of the 19th century when Herschel discovered the infrared radiation and Fraunhofer examined the spectral lines of the sun. These days, prism and first grating spectrometers have been developed and used.

In the 1970s a new technology for infrared spectroscopy came up, the Fourier-Transform-Spectroscopy. This technology uses an interferometer with a fixed and a moving mirror and allows the acquisition of spectra with high resolution and large spectral range.

The new technology, however, required a computer for the calculation of the spectrum. Therefore, a real breakthrough came only when personal computers got sufficient powerful in the 1990s.

Today, some other spectral technologies are being developed using e.g. tunable Fabry-Perot-Filters integrated with detector packages or Quantum Cascade Lasers. Tunable Fabry-Perot-Filters consist of two highly reflective mirrors which form a cavity. The width of this cavity can be changed to affect the transmission of these filters. Quantum Cascade Lasers are highly brilliant light sources which emit radiation in the mid IR spectral range. These lasers consist of multi-layer semiconductor structures forming quantum wells of different size. Current and temperature allow the tuning of the emitted wavelength over a small range. Quantum Cascade Lasers with external cavities can be tuned over a spectral range of up to $200 \mathrm{~cm}^{-1}$ or more.

However, the spectral range of these technologies is still limited and FTIR-spectroscopy with its high spectral resolution and wide bandwidth is still the most flexible tool for a large number of applications.

The principle operation of an FTIR-spectrometer is shown in figure 1. The light from a broadband IR source is collimated, normally using a parabolic mirror, and guided to a beamsplitter. Nominally $50 \%$ of the radiation is transmitted to a fixed mirror and $50 \%$ is reflected to a movable mirror. Both beams are then reflected by the respective mirrors, combined again at the beamsplitter and focussed onto a detector. The detector translates the interfering beams into an electrical signal which is processed by electronics.

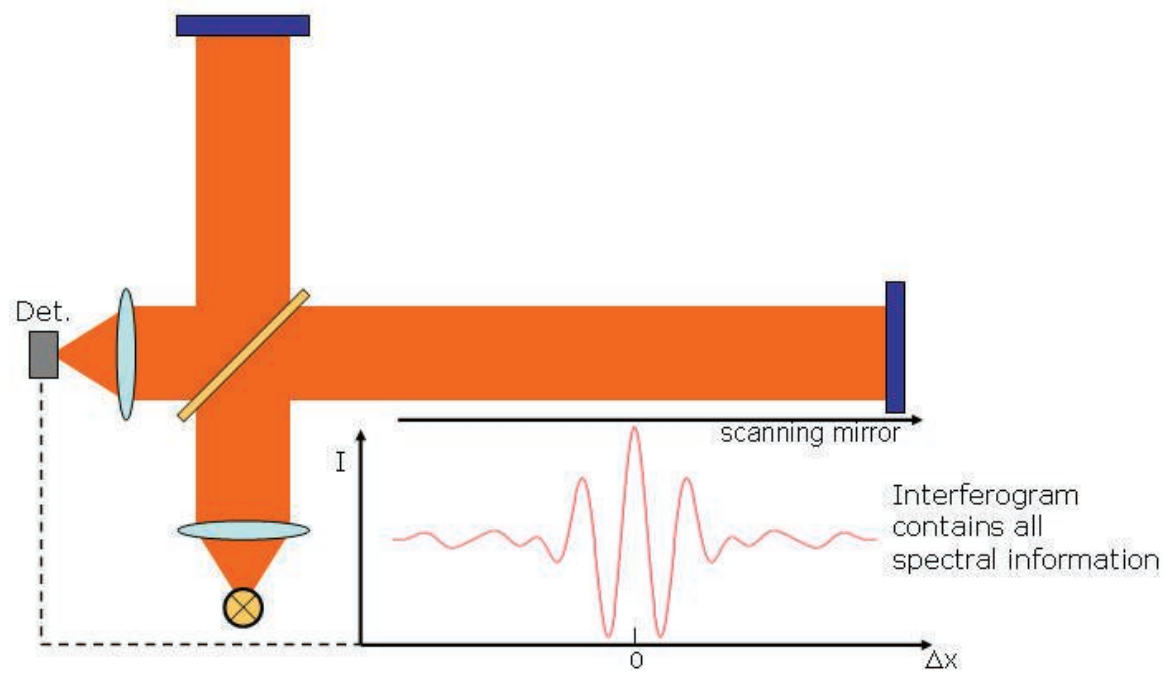

Figure 1: principle operation of a FTIR-spectrometer

One mirror of this interferometer is moving forth and back. The "interferogram" is acquired by recording the electrical signal of the detector as a function of the optical path difference. The interferogram contains all spectral information. A mathematical operation, the Fourier transformation, calculates the spectrum. Figure 2 shows interferogram and related spectrum. 
FT-IR spectroscopy is a single beam technique: A reference interferogram without and an interferogram with a sample in the beam are recorded, the corresponding spectra are divided by each other in order to receive a transmission spectrum of the sample.

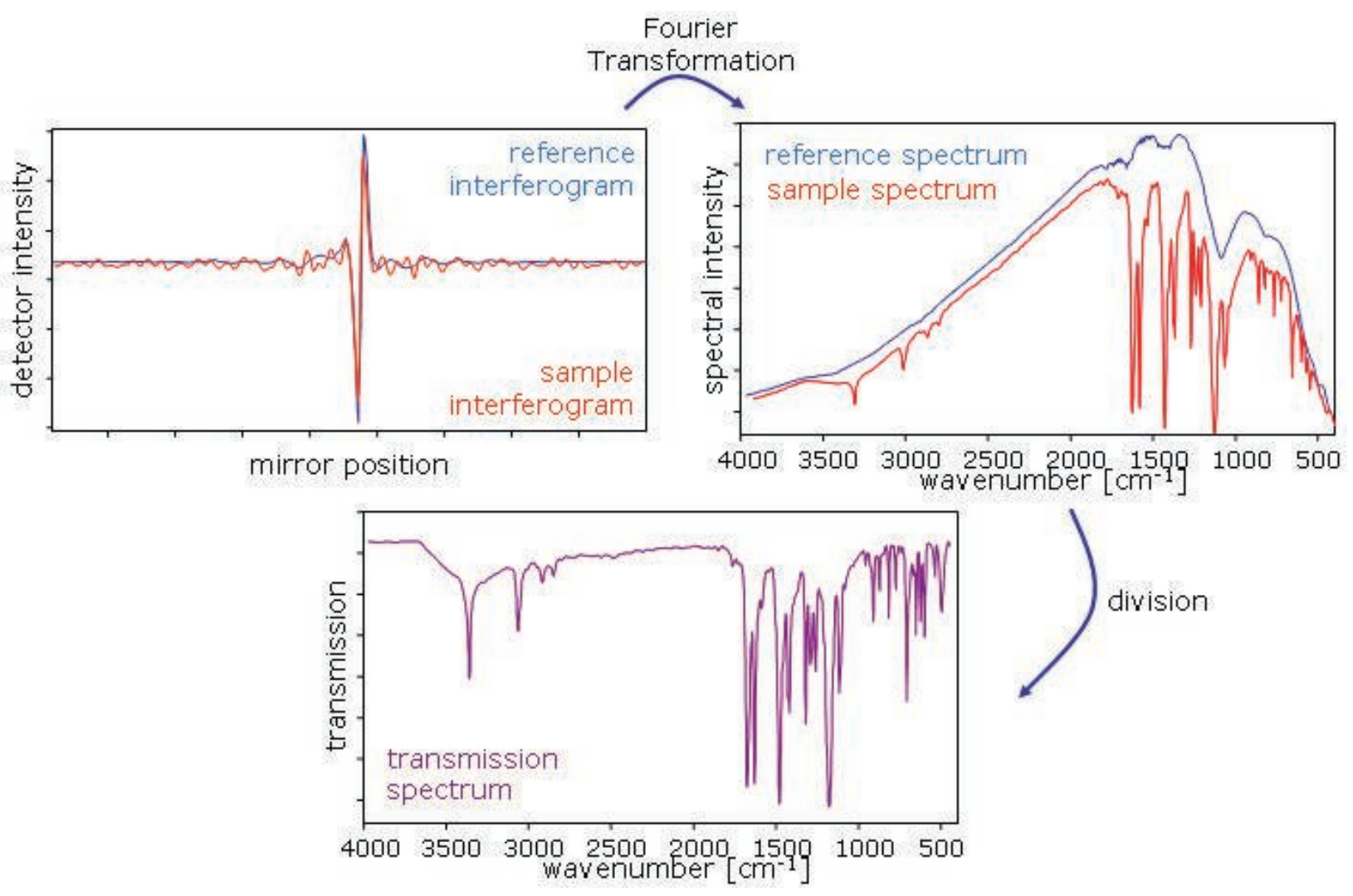

Figure 2: from the recorded interferogram to the spectrum

In comparison with a grating spectrometer a Fourier spectrometer has several advantages: The multiplex or Fellgett's advantage represents the fact that at each position of the scanning mirror signal from all wavelengths in the spectrum are recorded simultaneously. The second advantage is the Connes' advantage. As the position of the moving mirror is measured using the interferences of a laser, the spectrometer utilizes an integrated highly accurate wavelength/wavenumber reference. The third advantage is the throughput advantage. Where grating spectrometers need small rectangular entrance slits to achieve a desired spectral resolution, FTIR-spectrometers achieve similar spectral resolution using circular and most likely larger entrance apertures. A grating spectrometer with a focal length of $f=150 \mathrm{~mm}$, e.g., would need a slit of $11 \mu \mathrm{m}$ width to achieve a spectral resolution of $\Delta \lambda=0.4 \mathrm{~nm}$ at $2000 \mathrm{~nm}$. A FTIR system with the same focal length would achieve the same spectral resolution with a circular aperture of $4.2 \mathrm{~mm}$ diameter.

Early FT-IR spectrometers were large and bulky. Over the past 30 years, FTIR spectrometers could change from large systems operated by scientists to compact and easy to use systems (compare figure 3 ). This change was possible by the advances in computers, electronics and optical technologies.

Where first interferometers in the 1970s consisted of large optical elements today's interferometers can be built very compact, rugged and more accurate. An example is shown in figure 4 . The interferometer uses cube corner mirrors on a seesaw to achieve the optical path difference. Such interferometer designs allow the construction of very compact and robust spectrometers. And new designs using novel production technologies (MEMS) have been announced which could lead to even smaller analyzers.

Ultra high resolution systems, however, need longer path differences. These systems still require linear scanners. For these systems other concepts are available which ensure an accurate movement of the mirror over a long distance. Some systems utilize active compensation mirrors which balance possible 
tilts of the mirror. There are FT-spectrometers on the market which allow path differences in the interferometer of over $10 \mathrm{~m}$ which is required for ultra-high resolution spectroscopy of gases.
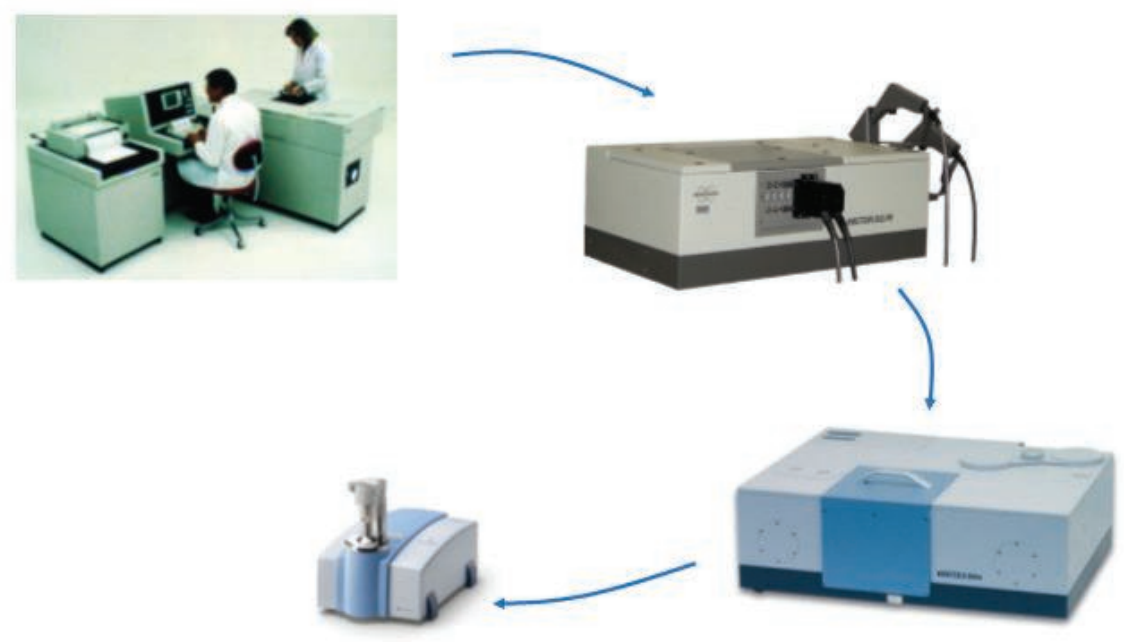

Figure 3: evolution of FTIR spectrometers in the past 30 years.

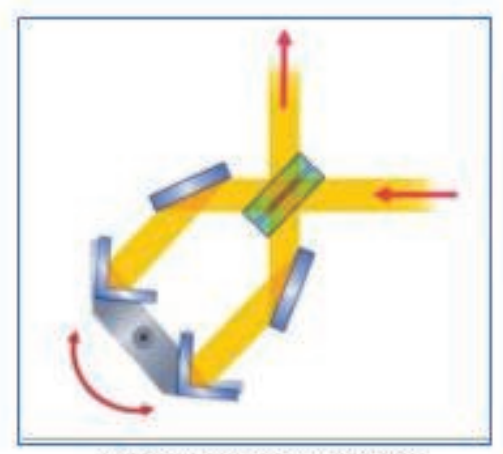

US Patent No 5.309 .217

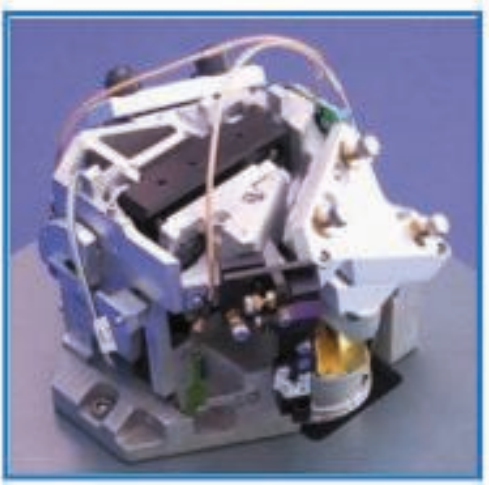

Figure 4: example of a compact interferometer using cube corner technology

The progress in computer and electronics notably the power of the PCs led to powerful and easy to use FT-IR spectrometers. The development of analogue to digital converters with high dynamic range and switched power supplies reduced the power consumption and size while increasing the performance of the spectrometers. The advances in optical communication, notably the development of optical fibers and sensitive detectors made it possible to develop NIR-analysers which are used in quality control and in process applications.

Today's FTIR-spectrometers cover a huge range of applications in research and in industrial applications. High resolution systems are used to monitor the composition of the atmosphere or for remote sensing of gases in the environment. Other systems find application in the quality control of industrial products. FTNIR-spectrometers are widely used for quality control in the food \& feed and for raw materials identification in the chemical and pharmaceutical industry. Advanced instrumentation is used for chemical imaging in analysing industrial samples or biological tissue.

Specially designed systems are used in the semiconductor industry for analysis of impurities in crystalline silicon in the 10ppta (parts per trillion) range. 
Some of these applications are presented in the following. Figure 5 shows the application of Raman Spectroscopy to the non-destructive analysis of art. For samples which show fluorescence NIR excitation and detection by FT-Raman is used. For less fluorescing samples and measurements with higher sensitivities dispersive Raman systems are utilized. In this example a dispersive Raman system is used to analyse the type of paint and varnish the artist used. This knowledge can be helpful for restoration of a piece of art or to distinguish a falsification from a true piece of art.

Two applications for quality control using NIR spectrometers are shown in Figure 6. On the left image a NIR spectrometer is placed above a conveyor band and NIR radiation is directed onto the salt taken from the mine. The reflected radiation contains information about the purity and the water content of the salt. The image on the right shows a spectrometer front end which guides via optical fibres radiation reflected from polystyrene in a production plant to a spectrometer which is placed somewhere else at the production site.

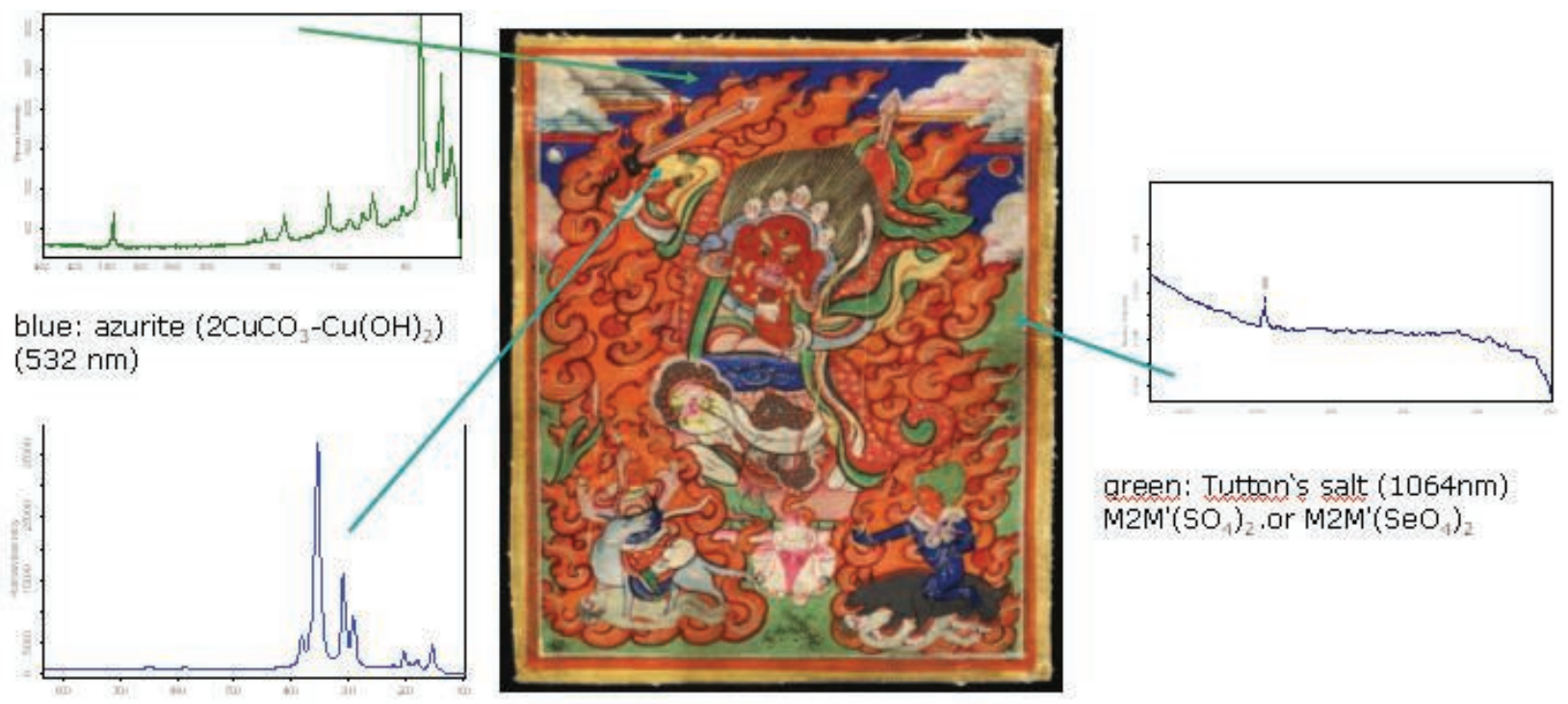

yellow: auripigment $\left(\mathrm{As}_{2} \mathrm{~S}_{3}\right)$

$(785 \mathrm{~nm})$

R. Ernst, ETH Zürich

Figure 5: Raman spectroscopy as a non-destructive tool for the analysis of art.

salt mine in $800 \mathrm{~m}$ depth !!!

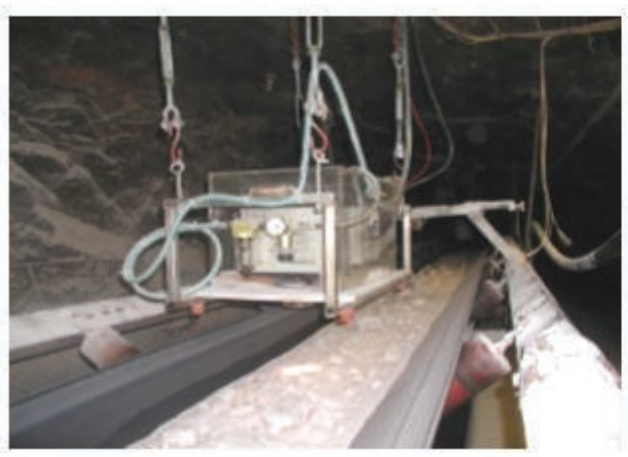

emission head

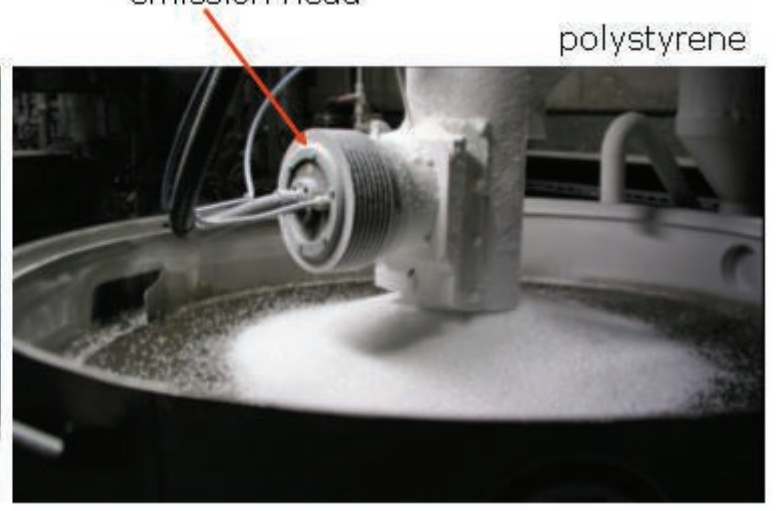

Figure 6: NIR spectroscopy for quality control in a salt mine (left) and during the production of polystyrene (right) 

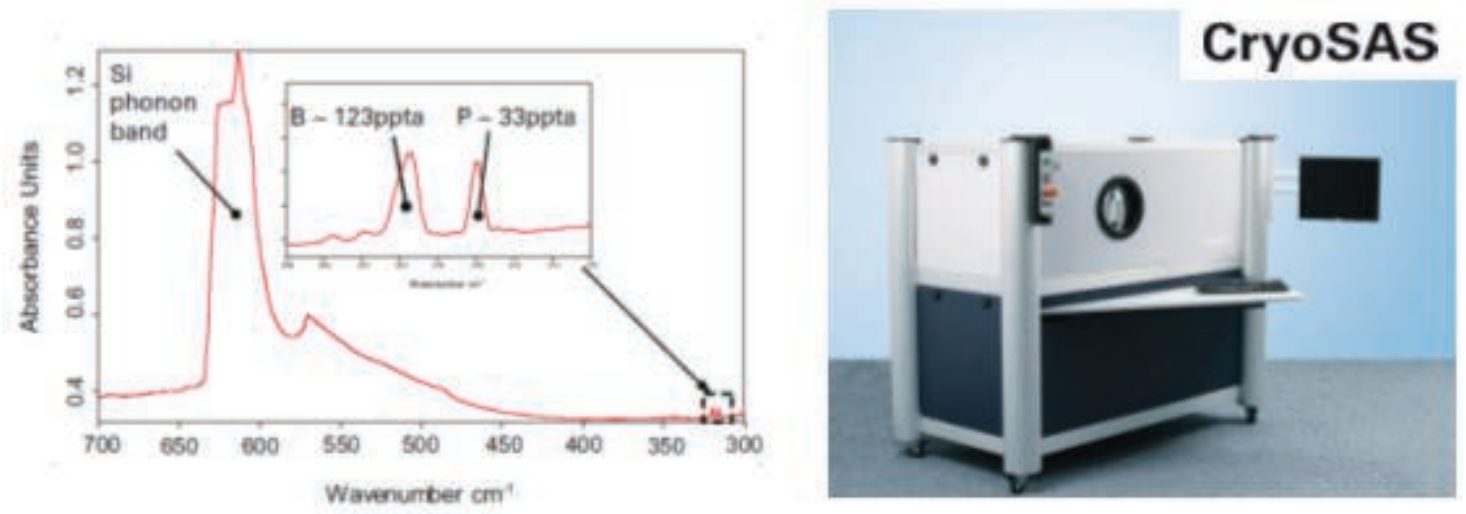

Figure 7: Far Infrared transmission spectrum of silicon at low temperatures. The cryogenic silicon analysis system is used to precisely determine the amount of impurities in crystalline silicon.

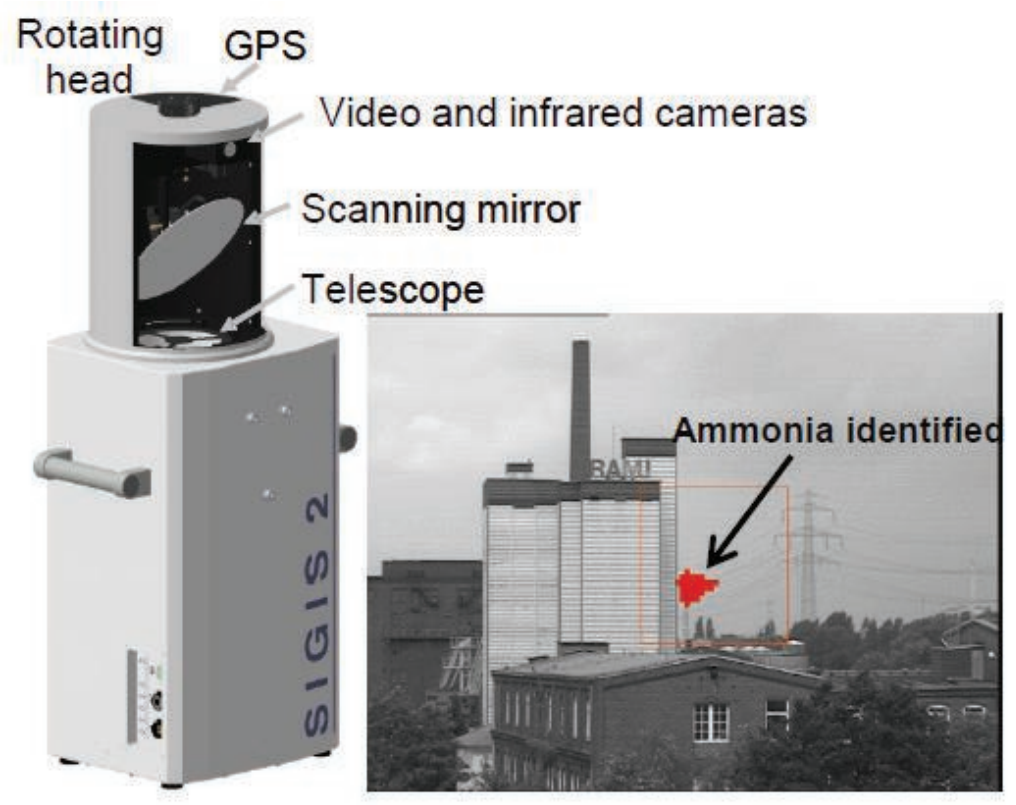

Figure 8: Remote detection system for detecting chemicals in clouds. On the right picture ammonia leaving a chimney is detected. 
Another application is shown in figure 7. Here, the spectrometer measures transmission spectra of crystalline silicon samples at temperatures around $\mathrm{T}=10 \mathrm{~K}$. The spectra are records in the range between $\lambda=15 \mu \mathrm{m}$ and $\lambda=30 \mu \mathrm{m}$. This kind of analysis allows the detection of impurities in crystalline silicon as low as 10 ppta (parts per trillion).

Fourier-Transform-Spectroscopy is also be used for remote detection of gases. In Figure 8 a spectrometer with a telescope and a scanning system is presented which uses the small temperature differences between gases and the environment to analyses gas clouds. The image on the right in figure 8 shows the identification of ammonia emitted from a smoke stack of an industrial site. 